Research Article

\title{
Higher-Order Compact Finite Difference for Certain PDEs in Arbitrary Dimensions
}

\author{
Yan Gao ${ }^{1}$ and Songlin Liu $\mathbb{D}^{2}$ \\ ${ }^{1}$ Department of Information Engineering, Wuhan Business University, Wuhan 430056, China \\ ${ }^{2}$ Faculty of Mathematics and Statistics, Key Laboratory of Applied Mathematics of Hubei Province, Hubei University, \\ Wuhan 430062, China
}

Correspondence should be addressed to Songlin Liu; liusonglin@hubu.edu.cn

Received 2 July 2020; Revised 30 August 2020; Accepted 5 October 2020; Published 24 November 2020

Academic Editor: Xinguang Zhang

Copyright (C) 2020 Yan Gao and Songlin Liu. This is an open access article distributed under the Creative Commons Attribution License, which permits unrestricted use, distribution, and reproduction in any medium, provided the original work is properly cited.

\begin{abstract}
In this paper, we first present the expression of a model of a fourth-order compact finite difference (CFD) scheme for the convection diffusion equation with variable convection coefficient. Then, we also obtain the fourth-order CFD schemes of the diffusion equation with variable diffusion coefficients. In addition, a fine description of the sixth-order CFD schemes is also developed for equations with constant coefficients, which is used to discuss certain partial differential equations (PDEs) with arbitrary dimensions. In this paper, various ways of numerical test calculations are prepared to evaluate performance of the fourth-order CFD and sixth-order CFD schemes, respectively, and the empirical results are proved to verify the effectiveness of the schemes in this paper.
\end{abstract}

\section{Introduction}

The standard strategy associated with generating higher order finite difference schemes to expand the stencil is proposed by Leonard [1]. The major disadvantage of these approaches is to widen the computational stencil with the increasing order of the approximation, leading to larger matrix bandwidths, which shall complicate the numerical analysis near the boundaries, while communication to implement on parallel computer architecture is raising demand. In consideration of the problems caused by noncompact finite difference methods, it is desirable to develop a class of schemes involving compact with high order.

In recent years, a great deal of efforts has been devoted to developing the designing schemes of CFD for solving various partial differential equations (see, e.g., [2-7]). A derivation of fourth-order and sixth-order compact difference schemes for the three dimensional Poisson equation is developed by Zhai and Feng [8] finite volume (FV) method, based on two different types of dual partitions. A new design of sixth-order compact finite-difference method with a nine-point stencil is developed by Nabavi et al. [9] to solve the Helmholtz equation in two-dimensional domain under the circumstance of Dirichlet and Neumann boundary. With the idea of the immersed interface method, third- and fourth-order compact finite difference schemes were proposed for solving the Helmholtz equations with discontinuous coefficient [10, $11]$. It is worth noting that the advert of $[12,13]$ a new high-order finite difference discretization strategy, based on the Richardson extrapolation technique and an operator interpolation scheme, is explored to solve convection diffusion equations [14] which exploits an innovative adaptive scheme in terms of Adaptive Mesh Refinement (AMR) and Multigrid Algorithms to achieve a settlement of the fourthorder two-dimensional Poisson equation. In addition, a great number of studies reported by Bilbao and Hamilton [15] provides a two-step schemes (which operate over three time levels) of higher order accurate finite difference schemes applied for the wave equation in any number of spatial dimensions.

The main aim of the present work is to provide a general formulation and simple approach of higher order CFD 
schemes for steady elliptic diffusion and convection-diffusion problems in any dimension. The primary concern of this paper focuses on the following convection-diffusion equation with Dirichlet boundary conditions

$$
\left\{\begin{array}{l}
-\Delta u+\boldsymbol{\alpha} \cdot \nabla u=f, \text { in } \Omega=[0,1]^{d}, \\
\left.u\right|_{\partial \Omega}=g
\end{array}\right.
$$

where $\boldsymbol{\alpha}=\left(\alpha_{1}, \cdots, \alpha_{d}\right)$ is the velocity vector with dimension $d$, the source term $f$ is known analytically throughout the domain $\Omega$, and $g$ is given on the boundary $\partial \Omega, \Delta u=\sum_{m=1}^{d}$ $u_{x_{m} x_{m}}$ is the Laplacian operator, and $\nabla u=\left(u_{x_{1}}, \cdots, u_{x_{d}}\right)$ is the divergence operator.

Obviously, it is not difficult to derive the fourth-order compact finite difference scheme, especially for the case when $\alpha$ is a constant vector. The scheme in response to simple procedure is introduced in Theorems 2 and 4 in the next section. The compact scheme of the sixth-order finite difference scheme for (1) with constant parameters will be completely described by Theorem 6 in Section 3.

The paper is organized as follows. In Section 2, the forthorder CFD schemes are derived for the convection-diffusion problems with variable convective coefficients in any dimension. Since then, two different fourth-order CFD schemes are linked to the consequent of steady elliptic diffusion equations with variable diffusive coefficients. The sixth-order CFD schemes are considered to be the solution of the convectiondiffusion problems with constant convective coefficients in Section 3. The analysis consisted of two numerical examples is presented to verify the feasibility and high-order accuracy of the proposed methods in Section 4. This paper concludes with a discussion in Section 5.

\section{Fourth-Order CFD Schemes for Convection- Diffusion Equations}

2.1. Fourth-Order CFD Schemes for Convection-Diffusion Equations with Variable Convective Coefficients. Let $\pi_{h}=$ $\left\{\left(x_{i_{1}}, \cdots, x_{i_{d}}\right): x_{i_{k}}=i_{k} h, i_{k}=0, \cdots, n+1, k=1, \cdots, d\right\}$ be a uniform partition of $\Omega$ with the step size $h=1 /(n+1)$. Moreover, we introduce the central difference approximations $\delta_{x_{m}} u$ and $\delta_{x_{m} x_{m}} u$ as the first and second derivatives, respectively, by using the immediate neighbours. Denote $u_{i_{1}, \cdots, i_{d}}$ by the value of $u\left(x_{1}, \cdots, x_{d}\right)$ at the nodes $\left(x_{i_{1}}, \cdots, x_{i_{d}}\right)$, and

$$
\begin{aligned}
\left.\delta_{x_{m}} u\right|_{\left(x_{i_{1}}, \cdots, x_{i_{d}}\right)} & =\frac{u_{i_{1}, \cdots, i_{m}+1, \cdots, i_{d}}-u_{i_{1}, \cdots, i_{m}-1, \cdots, i_{d}}}{2 h}, \\
\left.\delta_{x_{m} x_{m}} u\right|_{\left(x_{i_{1}}, \cdots, x_{i_{d}}\right)} & =\frac{u_{i_{1}, \cdots, i_{m}+1, \cdots, i_{d}}-2 u_{i_{1}, \cdots, i_{m}, \cdots, i_{d}}+u_{i_{1}, \cdots, i_{m}-1, \cdots, i_{d}}}{h^{2}},
\end{aligned}
$$

by the first order and second order central difference schemes, respectively, in the $x_{m}(m=1, \cdots, d)$ direction.
By using the Taylor expansion, the problem (1) can be approximated by

$$
\sum_{m=1}^{d}\left(-\delta_{x_{m} x_{m}}+\alpha_{m} \delta_{x_{m}}\right) u+\mathscr{R} u=f,
$$

where

$$
\mathscr{R} u=\frac{h^{2}}{6} \mathscr{R}^{(1)} u+O\left(h^{4}\right)
$$

with

$$
\mathscr{R}^{(1)} u=\sum_{m=1}^{d}\left(\frac{1}{2} u_{x_{m} x_{m} x_{m} x_{m}}-\alpha_{m} u_{x_{m} x_{m} x_{m}}\right)
$$

Remark 1. By neglecting $\mathscr{R} u$ in (4), we obtain the simplest CFD scheme, with a second order accuracy, for (1), given by

$$
\sum_{m=1}^{d}\left(-\delta_{x_{m} x_{m}} u+\alpha_{m} \delta_{x_{m}} u\right)=f
$$

which is a 5 -point scheme in $2 \mathrm{D}$ and a 7 -point one in $3 \mathrm{D}$.

To obtain higher order CFD schemes, we will approximate the high order partial differential terms in (4) in a compact stencil.

Theorem 2. For $\mathscr{R}^{(1)}$ u defined in (5), we have

$$
\begin{aligned}
\mathscr{R}^{(1)} u= & \frac{1}{2} \mathscr{F}_{1}-\frac{1}{2} \sum_{m=1}^{d} \sum_{n=1, n \neq m}^{d} \delta_{x_{m} x_{m}} \delta_{x_{n} x_{n}} u \\
& +\sum_{m=1}^{d} \alpha_{m} \sum_{n=1, n \neq m}^{d} \delta_{x_{m}} \delta_{x_{n} x_{n}} u-\sum_{m=1}^{d} a_{m m} \delta_{x_{m} x_{m}} u \\
& -\frac{1}{2} \sum_{m=1}^{d} \sum_{n=1, n \neq m}^{d} a_{m n} \delta_{x_{m}} \delta_{x_{n}} u-\sum_{m=1}^{d} a_{m} \delta_{x_{m}} u+O\left(h^{2}\right),
\end{aligned}
$$

where

$$
\begin{aligned}
\mathscr{F}_{1} & =\sum_{m=1}^{d}\left(-f_{x_{m} x_{m}}+\alpha_{m} f_{x_{m}}\right), \\
a_{m} & =\frac{1}{2}\left(-\Delta \alpha_{m}+\sum_{n=1}^{d} \alpha_{n}\left(\alpha_{m}\right)_{x_{n}}\right), \\
a_{m m} & =\frac{1}{2} \alpha_{m}^{2}-\left(\alpha_{m}\right)_{x_{m}}, \\
a_{m n} & =\alpha_{m} \alpha_{n}-\left(\alpha_{m}\right)_{x_{n}}-\left(\alpha_{n}\right)_{x_{m}}, m \neq n,
\end{aligned}
$$

and $\left(\alpha_{m}\right)_{x_{n}}$ denote the derivation of $\alpha_{m}$ along the $x_{n}-$ direction. 
Proof. Differentiating both sides of (1) with respect to the $m$ -th direction gives

$$
-\sum_{n=1}^{d} u_{x_{m} x_{n} x_{n}}+\sum_{n=1}^{d} \alpha_{n} u_{x_{m} x_{n}}+\sum_{n=1}^{d}\left(\alpha_{n}\right)_{x_{m}} u_{x_{n}}=f_{x_{m}} .
$$

Differentiating both sides of (10) respect to the $m$-th direction again and then using (10) gives

$$
\begin{aligned}
& -\sum_{n=1}^{d} u_{x_{m} x_{m} x_{n} x_{n}}+\sum_{n=1}^{d} \alpha_{n} u_{x_{m} x_{m} x_{n}} \\
& \quad+2 \sum_{n=1}^{d}\left(\alpha_{n}\right)_{x_{m}} u_{x_{m} x_{n}}+\sum_{n=1}^{d}\left(\alpha_{n}\right)_{x_{m} x_{m}} u_{x_{n}}=f_{x_{m} x_{m}} .
\end{aligned}
$$

Therefore, adding (11) with each direction, we get

$$
\begin{aligned}
\mathscr{R}^{(1)} u= & \frac{1}{2} \mathscr{F}_{1}-\frac{1}{2} \sum_{m=1}^{d} \sum_{n=1, n \neq m}^{d} u_{x_{m} x_{m} x_{n} x_{n}} \\
& -\sum_{m=1}^{d} \sum_{n=1, n \neq m}^{d} \alpha_{m} u_{x_{m} x_{n} x_{n}}-\sum_{m=1}^{d} a_{m m} u_{x_{m} x_{m}} \\
& -\frac{1}{2} \sum_{m=1}^{d} \sum_{n=1, n \neq m}^{d} a_{m n} u_{x_{m} x_{n}}-\sum_{m=1}^{d} a_{m} u_{x_{m}}
\end{aligned}
$$

where $\mathscr{F}_{1}$ appeared in (8). By using the central difference schemes

$$
\begin{gathered}
u_{x_{m}}=\delta_{x_{m}} u+O\left(h^{2}\right), \\
u_{x_{m} x_{m}}=\delta_{x_{m} x_{m}} u+O\left(h^{2}\right), \\
u_{x_{m} x_{n}}=\delta_{x_{m}} \delta_{x_{n}} u+O\left(h^{2}\right), \\
u_{x_{m} x_{n} x_{n}}=\delta_{x_{m}} \delta_{x_{n} x_{n}} u+O\left(h^{2}\right), \\
u_{x_{m} x_{m} x_{n} x_{n}}=\delta_{x_{m} x_{m}} \delta_{x_{n} x_{n}} u+O\left(h^{2}\right),
\end{gathered}
$$

with $n \neq m$, we can get (7), which completes the proof of Theorem 2 .

From equations (3), (4), (5), and Theorem 2, a fourthorder compact difference scheme for approximating (1) is obtained, which can be represented as follows

$$
\begin{aligned}
& \sum_{m=1}^{d} \sum_{\substack{n=1 \\
n \neq m}}^{d}\left(c_{m n} \delta_{x_{m}} \delta_{x_{n}}+c_{m n n} \delta_{x_{m}} \delta_{x_{n} x_{n}}+c_{m m n n} \delta_{x_{m} x_{m}} \delta_{x_{n} x_{n}}\right) u \\
& +\sum_{m=1}^{d}\left(c_{m} \delta_{x_{m}}+c_{m m} \delta_{x_{m} x_{m}}\right) u=-f+\frac{h^{2}}{12} \mathscr{F}_{1},
\end{aligned}
$$

where

$$
\begin{aligned}
& c_{m}=-\alpha_{m}+\frac{h^{2}}{6} a_{m}, \quad c_{m m}=1+\frac{h^{2}}{6} a_{m m}, \\
& c_{m n}=\frac{h^{2}}{6} a_{m n}, \quad c_{m n n}=-\frac{h^{2}}{6} \alpha_{m}, \quad c_{m m n n}=\frac{h^{2}}{6},
\end{aligned}
$$

and $a_{m}, a_{m m}, a_{m n}(m \neq n)$ are defined in (9).

Remark 3.

(i) If $d=2$, the scheme (14) is no other than one suggested by Gupta et al. [16], and if $d=3$, the scheme (14) is no other than the one suggested by Zhang [17].

(ii) If $\boldsymbol{\alpha}$ is a constant vector, it is easy to simplify the fourth-order CFD scheme (14) by reducing the terms (15) as

$$
\begin{aligned}
& c_{m}=-\alpha_{m}, \quad c_{m m}=1+\frac{h^{2}}{12} \alpha_{m}^{2}, \\
& c_{m n}=\frac{h^{2}}{6} \alpha_{m} \alpha_{n}, \quad c_{m n n}=-\frac{h^{2}}{6} \alpha_{m}, \quad c_{m m n n}=\frac{h^{2}}{6} \quad(m \neq n) .
\end{aligned}
$$

2.2. Fourth-Order CFD Schemes for Diffusion Equations with Variable Diffusive Coefficients. Consider the $d$-dimensional diffusion equation

$$
\left\{\begin{array}{l}
-\nabla \cdot(\kappa \nabla u)=\tilde{f}, \quad \text { in } \Omega \subset \mathbb{R}^{d} \\
\left.u\right|_{\partial \Omega}=\varphi
\end{array}\right.
$$

where the diffusive coefficient $\kappa$ is a scalar function which is assumed to be positive. It can be rewritten as

$$
-\sum_{n=1}^{d}\left(\kappa u_{x_{n} x_{n}}+\kappa_{x_{n}} u_{x_{n}}\right)=\tilde{f}
$$

Scheme I. By using the Taylor series expansion, the problem (17) can be approximated by

$$
-\sum_{m=1}^{d}\left(\kappa \delta_{x_{m} x_{m}}+\kappa_{x_{m}} \delta_{x_{m}}\right) u+\tilde{\mathscr{R}} u=\tilde{f} .
$$

$\tilde{\mathscr{R}} u$ is the same as (4) with different force terms, where

$$
\begin{aligned}
\tilde{\mathscr{R}} u & =\frac{h^{2}}{6} \tilde{\mathscr{R}}^{(1)} u+O\left(h^{4}\right), \\
\tilde{\mathscr{R}}^{(1)} u & =\sum_{m=1}^{d}\left(\frac{\kappa}{2} u_{x_{m} x_{m} x_{m} x_{m}}+\kappa_{x_{m}} u_{x_{m} x_{m} x_{m}}\right) .
\end{aligned}
$$


Theorem 4. For $\tilde{\mathscr{R}}^{(1)}$ u defined in (21), we have

$$
\begin{aligned}
\kappa \tilde{\mathscr{R}}^{(1)} u= & \sum_{m=1}^{d} \sum_{\substack{n=1 \\
n \neq m}}^{d}\left(-\frac{\kappa^{2}}{2} \delta_{x_{m} x_{m}} \delta_{x_{n} x_{n}}-\kappa \delta_{x_{m}} \delta_{x_{n} x_{n}}\right. \\
& \left.-\frac{1}{2} a_{m n} \delta_{x_{m}} \delta_{x_{n}}\right) u-\sum_{m=1}^{d} a_{m m} \delta_{x_{m} x_{m}} u \\
& -\sum_{m=1}^{d} a_{m} \delta_{x_{m}} u+\frac{1}{2} \tilde{\mathscr{F}}_{1}+O\left(h^{2}\right),
\end{aligned}
$$

where

$$
\begin{aligned}
& \tilde{\mathscr{F}}_{1}=\sum_{m=1}^{d}\left(-\kappa \tilde{f}_{x_{m} x_{m}}+\kappa_{x_{m}} \tilde{f}_{x_{m}}\right), \\
& a_{m}=\frac{1}{2} \sum_{n=1}^{d}\left(\kappa \kappa_{x_{m} x_{n} x_{n}}-\kappa_{x_{n}} \kappa_{x_{m} x_{n}}\right), \\
& a_{m m}=\kappa \kappa_{x_{m} x_{m}}-\kappa_{x_{m}}^{2}+\frac{1}{2} \kappa \sum_{n=1}^{d} \kappa_{x_{n} x_{n}}-\frac{1}{2} \sum_{n=1}^{d} \kappa_{x_{n}}^{2}, \\
& a_{m n}=2 \kappa \kappa_{x_{m} x_{n}}-\kappa_{x_{m}} \kappa_{x_{n}}, m \neq n .
\end{aligned}
$$

Proof. Differentiating both sides of (17) once and twice with respect to $x_{m}$, respectively, gives

$$
\begin{array}{r}
2 \kappa_{x_{m}} u_{x_{m} x_{m}}+\sum_{n=1}^{d}\left(\kappa u_{x_{n} x_{n} x_{m}}+\kappa_{x_{m} x_{n}} u_{x_{n}}\right) \\
+\sum_{\substack{n=1 \\
n \neq m}}^{d}\left(\kappa_{x_{m}} u_{x_{n} x_{m}}+\kappa_{x_{n}} u_{x_{m} x_{n}}\right)=-\tilde{f}_{x_{m}}, \\
3 \kappa_{x_{m}} u_{x_{m} x_{m} x_{m}}+\sum_{n=1}^{d}\left(\kappa u_{x_{m} x_{m} x_{n} x_{n}}+\kappa_{x_{m} x_{m} x_{n}} u_{x_{n}}\right) \\
+\sum_{\substack{n=1 \\
n \neq m}}^{d}\left(2 \kappa_{x_{m}} u_{x_{m} x_{n} x_{n}}+\kappa_{x_{m}} u_{x_{m} x_{m} x_{n}}\right. \\
\left.+\kappa_{x_{m} x_{m}} u_{x_{n} x_{n}}+2 \kappa_{x_{m} x_{n}} u_{x_{m} x_{n}}\right)=-\tilde{f}_{x_{m} x_{m}} .
\end{array}
$$

From (25), we can obtain the expression of $\kappa \kappa_{x_{m}} u_{x_{m} x_{m} x_{m}}$, and then, substituting (25) into (26) to eliminate the term concluding $u_{x_{m} x_{m} x_{m}}$ gives the expression of $\kappa^{2} u_{x_{m} x_{m} x_{m} x_{m}}$. By the definition of $\tilde{\mathscr{R}}^{(1)} u$, we have

$$
\begin{aligned}
\kappa \tilde{\mathscr{R}}^{(1)} u= & -\frac{\kappa^{2}}{2} \sum_{m=1}^{d} \sum_{\substack{n=1 \\
n \neq m}}^{d} u_{x_{m} x_{m} x_{n} x_{n}}-\kappa \sum_{m=1}^{d} \sum_{\substack{n=1 \\
n \neq m}}^{d} \kappa_{x_{m}} u_{x_{m} x_{n} x_{n}} \\
& -\sum_{m=1}^{d} a_{m m} u_{x_{m} x_{m}}-\frac{1}{2} \sum_{m=1}^{d} \sum_{\substack{n=1 \\
n \neq m}}^{d} a_{m n} u_{x_{m} x_{n}} \\
& -\sum_{m=1}^{d} a_{m} u_{x_{m}}+\frac{1}{2} \tilde{\mathscr{F}}_{1} .
\end{aligned}
$$

By using the central difference schemes defined in (13), we can get (22),which completes the proof of Theorem 4 .

From (19), (4), (21), and Theorem 4, a fourth-order compact difference scheme for approximating (17) is obtained by dropping off the higher order terms $O\left(h^{4}\right)$

$$
\begin{aligned}
& \sum_{m=1}^{d} c_{m} \delta_{x_{m}} u+\sum_{m=1}^{d} c_{m m} \delta_{x_{m} x_{m}} u+\sum_{m=1}^{d} \sum_{\substack{n=1 \\
n \neq m}}^{d} c_{m n} \delta_{x_{m}} \delta_{x_{n}} u \\
& +\sum_{m=1}^{d} \sum_{\substack{n=1 \\
n \neq m}}^{d} c_{m n n} \delta_{x_{m}} \delta_{x_{n} x_{n}} u+\sum_{m=1}^{d} \sum_{\substack{n=1 \\
n \neq m}}^{d} c_{m m n n} \delta_{x_{m} x_{m}} \delta_{x_{n} x_{n}} u=\tilde{\mathscr{F}},
\end{aligned}
$$

where

$$
\tilde{\mathscr{F}}=-\kappa \tilde{f}+\frac{h^{2}}{12} \tilde{\mathscr{F}}_{1},
$$

$$
\begin{aligned}
c_{m} & =\kappa \kappa_{x_{m}}+\frac{h^{2}}{6} a_{m}, \quad c_{m m}=\kappa^{2}+\frac{h^{2}}{6} a_{m m}, \\
c_{m n} & =\frac{h^{2}}{6} a_{m n}, \quad c_{m n n}=\frac{h^{2}}{6} \kappa \kappa_{x_{m}}, \quad c_{m m n n}=\frac{h^{2}}{12} \kappa^{2}, \quad m \neq n .
\end{aligned}
$$

$\tilde{\mathscr{F}}_{1}$ and $a_{m}, a_{m m}, a_{m n}(m \neq n)$ are defined by (23) and (24).

Scheme II. Now, we derive another fourth-order CFD scheme for approximating (17) based on the fourth-order CFD scheme for convection-diffusion equations discussed in Section 2.1. Since $\kappa$ is positive, we can also rewrite (17) as the form

$$
-\sum_{n=1}^{d}\left(u_{x_{n} x_{n}}+\frac{\kappa_{x_{n}}}{\kappa} u_{x_{n}}\right)=\frac{\tilde{f}}{\kappa} .
$$

Let $\boldsymbol{\alpha}=\left(\alpha_{1}, \cdots, \alpha_{d}\right)$ with $\alpha_{m}=-\kappa_{x_{m}} / \kappa(m=1, \cdots, d)$, then (31) has the same form of (1). Substituting this $\boldsymbol{\alpha}$ into (14) and (15) and then using some direct calculations, a new fourth-order compact finite difference scheme is obtained as 


$$
\begin{aligned}
\sum_{m=1}^{d} & \sum_{n=1}^{d}\left(c_{m n} \delta_{x_{m}} \delta_{x_{n}}+c_{m n n} \delta_{x_{m}} \delta_{x_{n} x_{n}}+c_{m m n n} \delta_{x_{m} x_{m}} \delta_{x_{n} x_{n}}\right) u \\
& +\sum_{m=1}^{d}\left(c_{m} \delta_{x_{m}}+c_{m m} \delta_{x_{m} x_{m}}\right) u=-\kappa \tilde{f}+\frac{h^{2}}{12} \tilde{\mathscr{F}}_{1}=\tilde{\mathscr{F}}
\end{aligned}
$$

where

$$
\begin{aligned}
\tilde{\mathscr{F}}= & \sum_{m=1}^{d}\left(\kappa^{2} \tilde{f}_{x_{m} x_{m}}-\kappa \kappa_{x_{m}} \tilde{f}_{x_{m}}+\left(\kappa_{x_{m}}^{2}-\kappa \kappa_{x_{m} x_{m}}\right) \tilde{f}\right), \\
c_{m}= & \kappa^{2} \kappa_{x_{m}}+\frac{h^{2}}{12}\left[\sum_{n=1}^{d}\left(\kappa^{2} \kappa_{x_{m} x_{n} x_{n}}+\kappa_{x_{m}} \kappa_{x_{n}}^{2}\right)-2 \kappa \kappa_{x_{m}} \kappa_{x_{m} x_{m}}\right. \\
& \left.-\kappa \sum_{n=1, n \neq m}^{d}\left(\kappa_{x_{m}} \kappa_{x_{n} x_{n}}+\kappa_{x_{n}} \kappa_{x_{m} x_{n}}\right)\right], \\
c_{m m}= & \kappa^{3}+\frac{h^{2}}{12} \kappa\left(2 \kappa \kappa_{x_{m} x_{m}}-\kappa_{x_{m}}^{2}\right), \\
c_{m n}= & \frac{h^{2}}{6} \kappa\left(2 \kappa \kappa_{x_{m} x_{n}}-\kappa_{x_{m}} \kappa_{x_{n}}\right), \\
c_{m n n}= & \frac{h^{2}}{6} \kappa^{2} \kappa_{x_{m}}, \quad c_{m m n n}=\frac{h^{2}}{6} \kappa^{3}(m \neq n) .
\end{aligned}
$$

\section{Remark 5.}

(i) In practice, we should approximate the factors related to $\kappa$ and $\tilde{f}$ with the accuracy $O\left(h^{2}\right)$ in (24), like $\kappa_{x_{m}}, \kappa_{x_{m} x_{m}}, \kappa_{x_{m} x_{n}}(m \neq n), \kappa_{x_{m} x_{m} x_{n}}(m \neq n)$, and $\tilde{f}_{x_{m}}$ ' $\tilde{f}_{x_{m} x_{m}}$. All of these can be approximated by the corresponding central difference scheme to meet the requirement.

(ii) To guarantee the accuracy of the schemes (28) and (32), we also need to approximate $\kappa_{x_{m}}$ and $\kappa_{x_{m} x_{m} x_{m}}$ with the fourth-order accuracy. For the purpose of this, the following approximation

$$
\begin{aligned}
\kappa^{\prime}(x)= & -\frac{1}{6 h}\left[\kappa(x+h)-8 \kappa\left(x+\frac{h}{2}\right)\right. \\
& \left.+8 \kappa\left(x-\frac{h}{2}\right)-\kappa(x-h)\right]+O\left(h^{4}\right), \\
\kappa^{\prime \prime \prime}(x)= & \frac{4}{h^{3}}\left[\kappa(x+h)-2 \kappa\left(x+\frac{h}{2}\right)\right. \\
& \left.+2 \kappa\left(x-\frac{h}{2}\right)-\kappa(x-h)\right]+O\left(h^{2}\right),
\end{aligned}
$$

will be introduced.

\section{Sixth-Order CFD Schemes for Convection- Diffusion Equations}

In the previous section, we have obtained the fourth-order CFD scheme for (1). Especially for the case that $\boldsymbol{\alpha}$ is a constant vector, this scheme seems very simple. Actually, after some further analysis, we can make it achieve higher order accuracy.

3.1. Sixth-Order CFD Schemes for Diffusion Equations with Variable Convection Coefficients. This study will offer a fresh insight into the following Theorem 6 to show the sixth-order CFD scheme for (1). By using the Taylor expansion, the problem (1) can be approximated by (3), where

$$
\mathscr{R} u=\frac{h^{2}}{6} \mathscr{R}^{(1)} u+\frac{h^{4}}{120} \mathscr{R}^{(2)} u+O\left(h^{6}\right),
$$

with

$$
\begin{aligned}
& \mathscr{R}^{(1)} u=\sum_{n=1}^{d}\left(\frac{1}{2} u_{x_{n} x_{n} x_{n} x_{n}}-\alpha_{n} u_{x_{n} x_{n} x_{n}}\right), \\
& \mathscr{R}^{(2)} u=\sum_{n=1}^{d}\left(\frac{1}{3} u_{x_{n} x_{n} x_{n} x_{n} x_{n} x_{n}}-\alpha_{n} u_{x_{n} x_{n} x_{n} x_{n} x_{n}}\right) .
\end{aligned}
$$

Theorem 6. Let $\mathscr{R} u$ be defined by (36), then we have

$$
\begin{aligned}
\mathscr{R} u= & \frac{h^{2}}{6} S_{1} u+\frac{h^{4}}{720}\left(S_{2}+S_{3}\right) u \\
& +\frac{h^{4}}{180}\left(S_{4}+S_{5}-S_{6}+S_{7}+S_{8}\right) u \\
& +\mathscr{F}^{*}+O\left(h^{6}\right),
\end{aligned}
$$

where

$$
\mathscr{F}^{*}=\frac{h^{2}}{12} \mathscr{F}_{1}+\frac{h^{4}}{720}\left(2 \mathscr{F}_{2}-\mathscr{F}_{3}-4 \mathscr{F}_{4}+4 \mathscr{F}_{5}-4 \mathscr{F}_{6}\right),
$$

with $S_{i} u(i=1, \cdots, 8)$ defined by (44), (59),(60), (65), (66), (71), (72), and (76) and $\mathscr{F}_{i}(i=1, \cdots, 6)$ defined by (45), (53), (61), (67), (73), and (77) below.

This study will offer the compact sixth-order finite difference scheme for (1)

$$
\sum_{m=1}^{d}\left(-\delta_{x_{m} x_{m}}+\alpha_{m} \delta_{x_{m}}\right) u+\mathscr{R} u=\mathscr{F}
$$

where $\mathscr{F}=f-\mathscr{F}^{*}$ and $\mathscr{R} U$ are defined in (39) by replacing the exact solution $u$ by the approximating solution $U$ and dropping off the remainder term $O\left(h^{6}\right)$. 

lows:

To prove Theorem 6, we define $Q_{i}(i=1,2, \cdots, 5)$ as fol-

$$
\begin{aligned}
& Q_{1}=\sum_{n=1}^{d} \alpha_{n}^{2} u_{x_{n} x_{n} x_{n} x_{n},} \\
& Q_{2}=2 \sum_{n=1}^{d} \sum_{\substack{k=1 \\
k \neq n}}^{d} \alpha_{n} \alpha_{k} u_{x_{n} x_{n} x_{n} x_{k}}, \\
& Q_{3}=2 \sum_{n=1}^{d} \sum_{\substack{k=1 \\
k \neq n}}^{d} \alpha_{k} u_{x_{n} x_{n} x_{n} x_{n} x_{k}} \\
& Q_{4}=2 \sum_{n=1}^{d} \sum_{\substack{k=1 \\
k \neq n}}^{d} \alpha_{k} u_{x_{n} x_{n} x_{k} x_{k} x_{k}} \\
& Q_{5}=2 \sum_{n=1}^{d} \sum_{\substack{k=1 \\
k \neq n}}^{d} u_{x_{n} x_{n} x_{n} x_{n} x_{k} x_{k}}
\end{aligned}
$$

and the following Lemmas 7-13 are needed.

Lemma 7. Let $\mathscr{R}^{(1)} u$ be defined in (37) and $Q_{k}(k=1, \cdots, 5)$ be defined in (42), then we have

$$
\begin{aligned}
\mathscr{R}^{(1)} u= & S_{1} u+\frac{h^{2}}{24}\left(Q_{1}+2 Q_{2}-Q_{3}-2 Q_{4}+Q_{5}\right) \\
& +\frac{1}{2} \mathscr{F}_{1}+O\left(h^{4}\right),
\end{aligned}
$$

where

$$
\begin{aligned}
& S_{1} u= \frac{1}{2} \sum_{n=1}^{d} \sum_{\substack{k=1 \\
k \neq n}}^{d}\left(-\delta_{x_{k} x_{k}} \delta_{x_{n} x_{n}}+\alpha_{k} \delta_{x_{k}} \delta_{x_{n} x_{n}}\right. \\
&\left.+\alpha_{n} \delta_{x_{k} x_{k}} \delta_{x_{n}}-\alpha_{n} \alpha_{k} \delta_{x_{k}} \delta_{x_{n}}\right) u-\frac{1}{2} \sum_{n=1}^{d} \alpha_{n}^{2} \delta_{x_{n} x_{n}} u, \\
& \mathscr{F}_{1}=\sum_{n=1}^{d}\left(-f_{x_{n} x_{n}}+\alpha_{n} f_{x_{n}}\right) .
\end{aligned}
$$

Proof. Differentiating both sides of (1) once and twice with respect to $x_{n}(n=1, \cdots, d)$, respectively, gives

$$
\begin{gathered}
-\sum_{k=1}^{d} u_{x_{k} x_{k} x_{n}}+\sum_{k=1}^{d} \alpha_{k} u_{x_{k} x_{n}}=f_{x_{n}}, \\
-\sum_{k=1}^{d} u_{x_{k} x_{k} x_{n} x_{n}}+\sum_{k=1}^{d} \alpha_{k} u_{x_{k} x_{n} x_{n}}=f_{x_{n} x_{n}},
\end{gathered}
$$

which leads to

$$
\begin{gathered}
u_{x_{n} x_{n} x_{n}}=-\sum_{\substack{k=1 \\
k \neq n}}^{d} u_{x_{k} x_{k} x_{n}}+\sum_{k=1}^{d} \alpha_{k} u_{x_{k} x_{n}}-f_{x_{n}}, \\
u_{x_{n} x_{n} x_{n} x_{n}}=-\sum_{\substack{k=1 \\
k \neq n}}^{d} u_{x_{k} x_{k} x_{n} x_{n}}+\sum_{k=1}^{d} \alpha_{k} u_{x_{k} x_{n} x_{n}}-f_{x_{n} x_{n}} .
\end{gathered}
$$

Using (47) and (48) to eliminate the terms $u_{x_{n} x_{n} x_{n}}$ and $u_{x_{n} x_{n} x_{n} x_{n}}$ in (37) yields

$$
\begin{aligned}
\mathscr{R}^{(1)} u= & -\frac{1}{2} \sum_{n=1}^{d} \sum_{\substack{k=1 \\
k \neq n}}^{d} u_{x_{k} x_{k} x_{n} x_{n}}+\frac{1}{2} \sum_{n=1}^{d} \sum_{\substack{k=1 \\
k \neq n}}^{d}\left(\alpha_{k} u_{x_{k} x_{n} x_{n}}+\alpha_{n} u_{x_{k} x_{k} x_{n}}\right) \\
& -\frac{1}{2} \sum_{n=1}^{d} \sum_{k=1}^{d} \alpha_{k} \alpha_{n} u_{x_{k} x_{n}}+\frac{1}{2} \sum_{n=1}^{d}\left(-f_{x_{n} x_{n}}+\alpha_{n} f_{x_{n}}\right) .
\end{aligned}
$$

Taking account of the centered difference approximations

$$
\begin{aligned}
u_{x_{n} x_{n}} & =\delta_{x_{n} x_{n}} u-\frac{h^{2}}{12} u_{x_{n} x_{n} x_{n} x_{n}}+O\left(h^{4}\right), \\
u_{x_{k} x_{n}} & =\delta_{x_{k}} \delta_{x_{n}} u-\frac{h^{2}}{6}\left(u_{x_{k} x_{k} x_{k} x_{n}}+u_{x_{k} x_{n} x_{n} x_{n}}\right)+O\left(h^{4}\right), \\
u_{x_{k} x_{k} x_{n}} & =\delta_{x_{k} x_{k}} \delta_{x_{n}} u-\frac{h^{2}}{12}\left(u_{x_{k} x_{k} x_{k} x_{k} x_{n}}+2 u_{x_{k} x_{k} x_{n} x_{n} x_{n}}\right)+O\left(h^{4}\right),
\end{aligned}
$$$$
u_{x_{k} x_{k} x_{n} x_{n}}=\delta_{x_{k} x_{k}} \delta_{x_{n} x_{n}} u-\frac{h^{2}}{12}\left(u_{x_{k} x_{k} x_{n} x_{n} x_{n} x_{n}}+u_{x_{k} x_{k} x_{k} x_{k} x_{n} x_{n}}\right)
$$$$
+O\left(h^{4}\right)
$$

with $n \neq k$, and noting the definition of $S_{1} u$ given by (44), then we have

$$
\begin{aligned}
\mathscr{R}^{(1)} u= & \frac{h^{2}}{24} \sum_{n=1}^{d} \sum_{\substack{k=1 \\
k \neq n}}^{d}\left[\left(u_{x_{k} x_{k} x_{n} x_{n} x_{n} x_{n}}+u_{x_{k} x_{k} x_{k} x_{k} x_{n} x_{n}}\right)\right. \\
& -\alpha_{k}\left(u_{x_{n} x_{n} x_{n} x_{n} x_{k}}+2 u_{x_{n} x_{n} x_{k} x_{k} x_{k}}\right) \\
& -\alpha_{n}\left(u_{x_{k} x_{k} x_{k} x_{k} x_{n}}+2 u_{x_{k} x_{k} x_{n} x_{n} x_{n}}\right) \\
& \left.+2 \alpha_{n} \alpha_{k}\left(u_{x_{k} x_{k} x_{k} x_{n}}+u_{x_{k} x_{n} x_{n} x_{n}}\right)\right] u \\
& +\frac{h^{2}}{24} \sum_{n=1}^{d} \alpha_{n}^{2} u_{x_{n} x_{n} x_{n} x_{n}}+S_{1} u+\frac{1}{2} \mathscr{F}_{1} \\
= & \frac{h^{2}}{24}\left(Q_{1}+2 Q_{2}-Q_{3}-2 Q_{4}+Q_{5}\right) \\
& +S_{1} u+\frac{1}{2} \mathscr{F}_{1}+O\left(h^{4}\right) .
\end{aligned}
$$


Therefore, the proof is completed when conditions (42) and (50) are satisfied.

Lemma 8. Let $\mathscr{R}^{(2)} u$ be defined by (38) and $Q_{k}(k=1, \cdots, 5)$ be defined by (42), then

$$
\mathscr{R}^{(2)} u=-\frac{1}{6}\left(4 Q_{1}+2 Q_{2}-Q_{3}-2 Q_{4}+Q_{5}\right)+\frac{1}{3} \mathscr{F}_{2},
$$

where

$$
\mathscr{F}_{2}=\sum_{n=1}^{d}\left(-f_{x_{n} x_{n} x_{n} x_{n}}+2 \alpha_{n} f_{x_{n} x_{n} x_{n}}\right) .
$$

Proof. Differentiating both sides of (1) three times and four times with respect to $x_{n}(n=1, \cdots, d)$, respectively, gives rise to

$$
\begin{aligned}
u_{x_{n} x_{n} x_{n} x_{n} x_{n}}= & -f_{x_{n} x_{n} x_{n}}-\sum_{\substack{k=1 \\
k \neq n}}^{d} u_{x_{k} x_{k} x_{n} x_{n} x_{n}}+\sum_{k=1}^{d} \alpha_{k} u_{x_{k} x_{n} x_{n} x_{n}}, \\
u_{x_{n} x_{n} x_{n} x_{n} x_{n} x_{n}}= & -f_{x_{n} x_{n} x_{n} x_{n}}+\sum_{\substack{k=1 \\
k \neq n}}^{d}\left(-u_{x_{k} x_{k} x_{n} x_{n} x_{n} x_{n}}\right. \\
& \left.+\alpha_{k} u_{x_{k} x_{n} x_{n} x_{n} x_{n}}\right)+\alpha_{n} u_{x_{n} x_{n} x_{n} x_{n} x_{n}} .
\end{aligned}
$$

Using (54) and (55) to eliminate the terms $u_{x_{n} x_{n} x_{n}}$ and $u_{x_{n} x_{n} x_{n} x_{n}}$ in (38) yields

$$
\begin{aligned}
\mathscr{R}^{(2)} u= & \frac{1}{3} \sum_{n=1}^{d} \sum_{\substack{k=1 \\
k \neq n}}^{d}\left(-u_{x_{n} x_{n} x_{n} x_{n} x_{k} x_{k}}+\alpha_{k} u_{x_{n} x_{n} x_{n} x_{n} x_{k}}\right. \\
& \left.+2 \alpha_{n} u_{x_{n} x_{n} x_{n} x_{k} x_{k}}-2 \alpha_{n} \alpha_{k} u_{x_{n} x_{n} x_{n} x_{k}}\right) \\
& -\frac{2}{3} \sum_{n=1}^{d} \alpha_{n}^{2} u_{x_{n} x_{n} x_{n} x_{n}}+\frac{1}{3} \mathscr{F}_{2} \\
= & -\frac{1}{6}\left(4 Q_{1}+2 Q_{2}-Q_{3}-2 Q_{4}+Q_{5}\right)+\frac{1}{3} \mathscr{F}_{2},
\end{aligned}
$$

which finishes the proof.

Lemma 9. Let $\mathscr{R} u$ be defined by (36) and $Q_{i}(i=1, \cdots, 5)$ be defined by (42), $S_{1} u, \mathscr{F}_{1}$, and $\mathscr{F}_{2}$ be defined by (44), (45), and (53) then

$$
\begin{aligned}
\mathscr{R} u= & \frac{h^{4}}{720} Q_{1}+\frac{h^{4}}{180}\left(2 Q_{2}-Q_{3}-2 Q_{4}+Q_{5}\right) \\
& +\frac{h^{2}}{6} S_{1} u+\frac{h^{2}}{6} \mathscr{F}_{1}+\frac{h^{4}}{360} \mathscr{F}_{2} .
\end{aligned}
$$

Proof. The proof can be easily done by putting Lemma 7 , Lemma 8., and the definition of $\mathscr{R} u$ together.
Now, we need to represent the terms $Q_{i}(i=1, \cdots, 5)$ by using compact finite difference approximations.

Lemma 10. Let $Q_{1}$ be defined by (42), then

$$
Q_{1}=S_{2} u+S_{3} u-\mathscr{F}_{3}+O\left(h^{2}\right),
$$

where

$$
S_{2} u=\sum_{n=1}^{d} \sum_{\substack{k=1 \\ k \neq n}}^{d} \alpha_{n}^{2}\left[\left(\alpha_{k} \delta_{x_{k}} \delta_{x_{n} x_{n}}-\alpha_{n} \delta_{x_{k} x_{k}} \delta_{x_{n}}\right)-\delta_{x_{k} x_{k}} \delta_{x_{n} x_{n}}\right] u,
$$

$$
\begin{aligned}
& S_{3} u=\sum_{n=1}^{d} \sum_{k=1}^{d} \alpha_{n}^{3} \alpha_{k} \delta_{x_{k} x_{n}} u, \\
& \mathscr{F}_{3}=\sum_{n=1}^{d} \alpha_{n}^{2}\left(f_{x_{n} x_{n}}+\alpha_{n} f_{x_{n}}\right) .
\end{aligned}
$$

Proof. Substituting (47) into (48) yields

$$
\begin{aligned}
u_{x_{n} x_{n} x_{n} x_{n}}= & \sum_{\substack{k=1 \\
k \neq n}}^{d}\left(-u_{x_{k} x_{k} x_{n} x_{n}}+\alpha_{k} u_{x_{k} x_{n} x_{n}}-\alpha_{n} u_{x_{k} x_{k} x_{n}}\right) \\
& +\alpha_{n} \sum_{k=1}^{d} \alpha_{k} u_{x_{k} x_{n}}-\left(f_{x_{n} x_{n}}+\alpha_{n} f_{x_{n}}\right) .
\end{aligned}
$$

Multiplying both sides of the above equation by $\alpha_{n}$ and then summing them over $n$, we can rewrite $Q_{1}$ defined in (42) as the form

$$
\begin{aligned}
Q_{1}= & \sum_{n=1}^{d} \alpha_{n}^{2} \sum_{\substack{k=1 \\
k \neq n}}^{d}\left[\left(\alpha_{k} u_{x_{k} x_{n} x_{n}}-\alpha_{n} u_{x_{k} x_{k} x_{n}}\right)-u_{x_{k} x_{k} x_{n} x_{n}}\right] \\
& +\sum_{n=1}^{d} \alpha_{n}^{2} \sum_{k=1}^{d} \alpha_{k} \alpha_{n} u_{x_{k} x_{n}}-\sum_{n=1}^{d} \alpha_{n}^{2}\left(f_{x_{n} x_{n}}+\alpha_{n} f_{x_{n}}\right) .
\end{aligned}
$$

Therefore, the expected result can be easily obtained by replacing the central difference approximations (13) in (63).

Lemma 11. Let $Q_{2}$ be defined by (42), then we have

$$
Q_{2}=S_{4} u+S_{5} u-\mathscr{F}_{4}+O\left(h^{2}\right),
$$

where

$$
S_{4} u=\sum_{n=1}^{d} \sum_{\substack{k=1 \\ k \neq n}}^{d} \alpha_{n}^{2} \alpha_{k} \delta_{x_{n} x_{n}} \delta_{x_{k}} u,
$$




$$
\begin{aligned}
S_{5} u= & \frac{1}{2} \sum_{n=1}^{d} \sum_{\substack{k=1 \\
k \neq n}}^{d} \sum_{\substack{m \neq 1 \\
m \neq n, m \neq k}}^{d}\left(-\alpha_{k} \alpha_{m} \delta_{x_{n} x_{n}} \delta_{x_{k}} \delta_{x_{m}}\right. \\
& \left.+\alpha_{n} \alpha_{k} \alpha_{m} \delta_{x_{n}} \delta_{x_{k}} \delta_{x_{m}}\right) u, \\
\mathscr{F}_{4}= & \frac{1}{2} \sum_{n=1}^{d} \sum_{\substack{k=1 \\
k \neq n}}^{d} \alpha_{n} \alpha_{k} f_{x_{n} x_{k}} .
\end{aligned}
$$

Proof. Differentiating both sides of (1) with respect to $x_{m} x_{k}$ $, k \neq m,(m, k=1, \cdots, d)$ yields

$$
\begin{aligned}
& -\sum_{n=1}^{d} \sum_{\substack{k=1 \\
k \neq n}}^{d} u_{x_{n} x_{n} x_{n} x_{k}}-\frac{1}{2} \sum_{n=1}^{d} \sum_{\substack{k=1 \\
k \neq n}}^{d} \sum_{\substack{m=1 \\
m \neq n, m \neq k}}^{d} u_{x_{n} x_{n} x_{m} x_{k}} \\
& \quad+\sum_{n=1}^{d} \sum_{\substack{k=1 \\
k \neq n}}^{d} \alpha_{n} u_{x_{n} x_{n} x_{k}}+\frac{1}{2} \sum_{n=1}^{d} \sum_{\substack{k=1 \\
k \neq n}}^{d} \sum_{\substack{m=1 \\
m \neq n, m \neq k}}^{d} \alpha_{n} u_{x_{n}} u_{x_{k}} u_{x_{m}} \\
& =\frac{1}{2} \sum_{m=1}^{d} \sum_{\substack{k=1 \\
k \neq m k \neq m}}^{d} f_{x_{m} x_{k}} .
\end{aligned}
$$
yields

Multiplying both sides of the above equation by $\alpha_{m} \alpha_{k}$

$$
\begin{aligned}
Q_{2}= & -\frac{1}{2} \sum_{n=1}^{d} \sum_{\substack{k=1 \\
k \neq n}}^{d} \sum_{\substack{m=1 \\
m \neq n, m \neq k}}^{d} \alpha_{m} \alpha_{k} u_{x_{n} x_{n} x_{m} x_{k}} \\
& +\frac{1}{2} \sum_{n=1}^{d} \sum_{\substack{k=1 \\
k \neq n}}^{d} \sum_{\substack{m=1 \\
m \neq n, m \neq k}}^{d} \alpha_{n} \alpha_{k} \alpha_{m} u_{x_{n}} u_{x_{k}} u_{x_{m}} \\
& +\sum_{n=1}^{d} \sum_{\substack{k=1 \\
k \neq n}}^{d} \alpha_{n}^{2} \alpha_{k} u_{x_{n} x_{n} x_{k}}-\frac{1}{2} \sum_{m=1}^{d} \sum_{\substack{k=1 \\
k \neq m}}^{d} \alpha_{m} \alpha_{k} f_{x_{m} x_{k}} .
\end{aligned}
$$

By using the centered difference approximations (13), we can obtain the results.

Lemma 12. Let $Q_{2}, Q_{3}$, and $Q_{4}$ be defined in (42), then we have

$$
Q_{2}-Q_{3}-Q_{4}=-S_{6} u+S_{7} u+\mathscr{F}_{5}+O\left(h^{2}\right)
$$

where

$$
\begin{aligned}
S_{6} u= & \sum_{n=1}^{d} \sum_{\substack{k=1 \\
k \neq n}}^{d} \sum_{\substack{m=1 \\
m \neq n, m \neq k}}^{d}\left[\left(\alpha_{k} \delta_{x_{k}} \delta_{x_{n} x_{n}}+\alpha_{n} \delta_{x_{n}} \delta_{x_{k} x_{k}}\right) \delta_{x_{m} x_{m}}\right. \\
& \left.-\alpha_{m}\left(\alpha_{k} \delta_{x_{n} x_{n}} \delta_{x_{k}}+\alpha_{n} \delta_{x_{n}} \delta_{x_{k} x_{k}}\right) \delta_{x_{m}}\right] u,
\end{aligned}
$$

$$
\begin{aligned}
& S_{7} u=2 \sum_{n=1}^{d} \sum_{\substack{k=1 \\
k \neq n}}^{d} \alpha_{n}^{2} \delta_{x_{n} x_{n}} \delta_{x_{k} x_{k}} u, \\
& \mathscr{F}_{5}=\sum_{n=1}^{d} \sum_{\substack{k=1 \\
k \neq n}}^{d}\left(\alpha_{k} f_{x_{n} x_{n} x_{k}}+\alpha_{n} f_{x_{n} x_{k} x_{k}}\right) .
\end{aligned}
$$

Proof. By using the definitions of $Q_{2}, Q_{3}$, and $Q_{4}$ in (42), we have

$$
\begin{aligned}
Q_{3}+Q_{4}= & \sum_{n=1}^{d} \sum_{\substack{k=1 \\
k \neq n}}^{d}\left[\alpha_{k}\left(u_{x_{n} x_{n}}+u_{x_{k} x_{k}}\right)_{x_{n} x_{n} x_{k}}\right. \\
& \left.+\alpha_{n}\left(u_{x_{n} x_{n}}+u_{x_{k} x_{k}}\right)_{x_{n} x_{k} x_{k}}\right] \\
= & -\mathscr{F}_{5}+Q_{2}+\sum_{n=1}^{d} \sum_{\substack{k=1 \\
k \neq n}}^{d}\left(\alpha_{k}^{2}+\alpha_{n}^{2}\right) u_{x_{n} x_{n} x_{k} x_{k}} \\
& +\sum_{n=1}^{d} \sum_{\substack{k=1 \\
k \neq n}}^{d} \sum_{\substack{m \neq 1 \\
m \neq n, m \neq k}}^{d} \alpha_{m}\left(\alpha_{k} u_{x_{m} x_{n} x_{n} x_{k}}+\alpha_{n} u_{x_{m} x_{n} x_{k} x_{k}}\right) \\
& -\sum_{n=1}^{d} \sum_{\substack{k=1 \\
k \neq n}}^{d} \sum_{\substack{m \neq 1 \\
m \neq n, m \neq k}}^{d}\left(\alpha_{k} u_{x_{m} x_{m} x_{n} x_{n} x_{k}}+\alpha_{n} u_{x_{m} x_{m} x_{n} x_{k} x_{k}}\right) .
\end{aligned}
$$

By using the centered difference approximations (13), we can obtain the results.

Lemma 13. Let $Q_{4}$ and $Q_{5}$ be defined in (42), then we have

$$
Q_{5}-Q_{4}=S_{8} u-\mathscr{F}_{6}+O\left(h^{2}\right)
$$

where

$$
\begin{aligned}
S_{8} u= & \sum_{n=1}^{d} \sum_{\substack{k=1 \\
k \neq n}}^{d} \sum_{\substack{m=1 \\
m \neq n, m \neq k}}^{d}\left(\alpha_{m} \delta_{x_{m}} \delta_{x_{n} x_{n}} \delta_{x_{k} x_{k}}\right. \\
& \left.-\delta_{x_{m} x_{m}} \delta_{x_{n} x_{n}} \delta_{x_{k} x_{k}}\right) u,
\end{aligned}
$$

$$
\mathscr{F}_{6}=\sum_{n=1}^{d} \sum_{\substack{k=1 \\ k \neq n}}^{d} f_{x_{n} x_{n} x_{k} x_{k}} .
$$

Proof. Differentiating both sides of (1) twice with respect to $x_{n}$ and then twice with respect to $x_{k}, k \neq n$, and then summing them over $n$ and $k$ gives 


$$
\begin{aligned}
& \sum_{n=1}^{d} \sum_{\substack{k=1 \\
k \neq n}}^{d} \sum_{m=1}^{d}\left(-u_{x_{m} x_{m} x_{n} x_{n} x_{k} x_{k}}+\alpha_{m} u_{x_{m} x_{n} x_{n} x_{k} x_{k}}\right) \\
& \quad=\sum_{n=1}^{d} \sum_{\substack{k=1 \\
k \neq n}}^{d} f_{x_{n} x_{n} x_{k} x_{k}},
\end{aligned}
$$

which can be rewritten as

$$
\begin{aligned}
& \sum_{n=1}^{d} \sum_{\substack{k=1 \\
k \neq n}}^{d}\left(-u_{x_{n} x_{n} x_{n} x_{n} x_{k} x_{k}}+\alpha_{n} u_{x_{n} x_{n} x_{n} x_{k} x_{k}}\right. \\
& \left.\quad-u_{x_{k} x_{k} x_{n} x_{n} x_{k} x_{k}}+\alpha_{k} u_{x_{k} x_{n} x_{n} x_{k} x_{k}}\right)+\sum_{n=1}^{d} \sum_{\substack{k=1 \\
k \neq n}}^{d} \sum_{\substack{m \neq n \\
m \neq n, m \neq k}}^{d} \\
& \cdot\left(-u_{x_{m} x_{m} x_{n} x_{n} x_{k} x_{k}}+\alpha_{m} u_{x_{m} x_{n} x_{n} x_{k} x_{k}}\right) \\
& =\sum_{n=1}^{d} \sum_{\substack{k=1 \\
k \neq n}}^{d} f_{x_{n} x_{n} x_{k} x_{k}} \cdot
\end{aligned}
$$
have

By using the definition of $Q_{4}$ and $Q_{5}$ defined in (42), we

$$
\begin{aligned}
Q_{5}-Q_{4}= & \sum_{n=1}^{d} \sum_{\substack{k=1 \\
k \neq n}}^{d} \sum_{\substack{m=1 \\
m \neq n, m \neq k}}^{d}\left(\alpha_{m} u_{x_{m} x_{n} x_{n} x_{k} x_{k}}\right. \\
& \left.-u_{x_{m} x_{m} x_{n} x_{n} x_{k} x_{k}}\right)-\sum_{n=1}^{d} \sum_{\substack{k=1 \\
k \neq n}}^{d} f_{x_{n} x_{n} x_{k} x_{k}}
\end{aligned}
$$

By using the centered difference approximations (13),we can obtain the results.

Proof of Theorem 6. The proof can be easily done by putting Lemmas 9-13 and the definition of $\mathscr{F}^{*}$ in (40) together.

3.2. Sixth-Order CFD Schemes for Diffusion Equations with Constant Convective Coefficients. It should be noted that the sixth-order CFD scheme given in (41) is valid for any dimension. For simplicity, we write down here the most commonly used schemes for (1), i.e., for 2 and 3 dimensions, in a concise way.

(i) If $d=2$, the terms including the summation symbol $\sum_{n=1}^{d} \sum_{k=1 k \neq n}^{d} \sum_{m=1 m \neq n, m \neq k}^{d}$ in $S_{i},(i=1, \cdots, 8)$, i.e., $S_{5}$, $S_{6}, S_{7}$, and $S_{8}$, vanish. Therefore, the sixth-order CFD scheme can be described as

$$
\begin{aligned}
& \sum_{n=1}^{2} \sum_{\substack{k=1 \\
k \neq n}}^{2}\left(c_{n k} \delta_{x_{n}} \delta_{x_{k}}+c_{n k k} \delta_{x_{n}} \delta_{x_{k} x_{k}}+c_{n n k k} \delta_{x_{n} x_{n}} \delta_{x_{k} x_{k}}\right) u \\
& +\sum_{n=1}^{2} c_{n} \delta_{x_{n}} u+\sum_{n=1}^{2} c_{n n} \delta_{x_{n} x_{n}} u=f-\mathscr{F}^{*}
\end{aligned}
$$

where

$$
\begin{aligned}
c_{n} & =\alpha_{n}, \\
c_{n n} & =1+\frac{\alpha_{n}^{2} h^{2}}{12}\left(1-\frac{\alpha_{n}^{2} h^{2}}{60}\right), \\
c_{n k} & =\frac{\alpha_{n} \alpha_{k} h^{2}}{12}\left(1-\frac{\left(\alpha_{n}^{2}+\alpha_{k}^{2}\right) h^{2}}{120}\right), \\
c_{n k k} & =\frac{\alpha_{n} h^{2}}{6}\left(1-\frac{\left(\alpha_{n}^{2}-9 \alpha_{k}^{2}\right) h^{2}}{120}\right), \\
c_{n n k k} & =\frac{h^{2}}{12}+\frac{\left(\alpha_{n}^{2}+\alpha_{k}^{2}\right) h^{4}}{160},
\end{aligned}
$$

and $\mathscr{F}^{*}$ appeared in (40).

(ii) If $d=3$, all of the $S_{i},(i=1, \cdots, 8)$ exist, and the sixthorder CFD scheme can be described as

$$
\begin{aligned}
& \sum_{\substack{n=1 \\
k}}^{3} \sum_{\substack{k \neq 1 \\
k \neq n}}^{3}\left(c_{n k} \delta_{x_{n}} \delta_{x_{k}}+c_{n k k} \delta_{x_{n}} \delta_{x_{k} x_{k}}+c_{n n k k} \delta_{x_{n}} \delta_{x_{k} x_{k}}\right) u \\
& \quad+\sum_{n=1}^{3}\left(a_{n} \delta_{x_{n}}+a_{n n} \delta_{x_{n} x_{n}}\right) u+\left(c_{123} \delta_{x_{1}} \delta_{x_{2}} \delta_{x_{3}}\right. \\
& \left.\quad+c_{112233} \delta_{x_{1} x_{1}} \delta_{x_{2} x_{2}} \delta_{x_{3} x_{3}}\right) u+\left(c_{1123} \delta_{x_{1} x_{1}} \delta_{x_{2}} \delta_{x_{3}}\right. \\
& \left.\quad+c_{1223} \delta_{x_{1}} \delta_{x_{2} x_{2}} \delta_{x_{3}}+c_{1233} \delta_{x_{1}} \delta_{x_{2}} \delta_{x_{3} x_{3}}\right) u \\
& \quad+\left(c_{12233} \delta_{x_{1}} \delta_{x_{2} x_{2}} \delta_{x_{3} x_{3}}+c_{11233} \delta_{x_{1} x_{1}} \delta_{x_{2}} \delta_{x_{3} x_{3}}\right. \\
& \left.\quad+c_{11223} \delta_{x_{1} x_{1}} \delta_{x_{2} x_{2}} \delta_{x_{3}}\right) u=f-\mathscr{F}^{*}
\end{aligned}
$$

where the forms of $c_{n}, c_{n n}, c_{n k}, c_{n k k}, c_{n n k k}$, and $k \neq n$ are the same as that defined in (82), except in this case $n=1,2,3$ and

$$
\begin{aligned}
c_{123} & =\frac{\alpha_{1} \alpha_{2} \alpha_{3} h^{4}}{30}, \\
c_{112233} & =\frac{h^{4}}{30}, \\
c_{m n k k} & =\frac{\alpha_{m} \alpha_{n} h^{4}}{90}, \\
c_{\text {mnnkk }} & =\frac{\alpha_{m} h^{4}}{90},
\end{aligned}
$$

where $m, n, k=1,2,3$, but they are different from each other.

\section{Numerical Results}

The CFD schemes derived in Section 2.1 for $2 \mathrm{D}$ and $3 \mathrm{D}$ convection-diffusion equations are discussed in literature $[16,17]$, respectively, and the corresponding numerical results have been completed to established. Hence, two examples in this filed are shown to validate the accuracy of the CFD schemes proposed in Section 2.2 and Section 3.2. 
TABLE 1: Numerical results for the fourth-order CFD method in 2D.

\begin{tabular}{|c|c|c|c|c|c|c|c|c|}
\hline \multirow{3}{*}{$h$} & \multicolumn{4}{|c|}{ Scheme I } & \multicolumn{4}{|c|}{ Scheme II } \\
\hline & \multicolumn{2}{|c|}{ Case 1} & \multicolumn{2}{|c|}{ Case 2} & \multicolumn{2}{|c|}{ Case 1} & \multicolumn{2}{|c|}{ Case 2} \\
\hline & Error & Ratio & Error & Ratio & Error & Ratio & Error & Ratio \\
\hline $1 / 4$ & $6.613 E-03$ & & $1.279 E-03$ & & $6.613 E-03$ & & $1.270 E-03$ & \\
\hline $1 / 8$ & $3.995 E-04$ & 4.04 & $8.355 E-05$ & 3.93 & $3.994 E-04$ & 4.04 & $8.341 E-05$ & 3.92 \\
\hline $1 / 16$ & $2.557 E-05$ & 3.96 & $5.273 E-06$ & 3.98 & $2.556 E-05$ & 3.96 & $5.271 E-06$ & 3.98 \\
\hline $1 / 32$ & $1.594 E-06$ & 4.00 & $3.330 E-07$ & 3.98 & $1.594 E-06$ & 4.00 & $3.330 E-07$ & 3.98 \\
\hline $1 / 64$ & $9.958 E-08$ & 4.00 & $2.082 E-08$ & 3.99 & $9.958 E-06$ & 4.00 & $2.082 E-08$ & 3.99 \\
\hline
\end{tabular}

TABLE 2: Numerical results for the fourth-order CFD method in 3D.

\begin{tabular}{llccccrrr}
\hline \multicolumn{2}{c}{ Case 1 } & \multicolumn{2}{c}{ Scheme I } & \multicolumn{2}{c}{ Case 1 } & \multicolumn{2}{c}{ Scheme II } & Case 2 \\
& Error & Ratio & Error & Ratio & Error & Ratio & Error & Ratio \\
\hline $1 / 4$ & $1.294 E-02$ & & $4.226 E-03$ & & $1.294 E-02$ & & $4.211 E-03$ \\
$1 / 8$ & $7.523 E-04$ & 4.10 & $2.560 E-04$ & 4.04 & $7.524 E-04$ & 4.10 & $2.558 E-04$ & 4.04 \\
$1 / 16$ & $4.740 E-05$ & 3.98 & $1.587 E-05$ & 4.01 & $4.740 E-05$ & 3.98 & $1.587 E-05$ & 4.01 \\
$1 / 32$ & $2.954 E-06$ & 4.00 & $9.898 E-07$ & 4.00 & $2.955 E-06$ & 4.00 & $9.898 E-07$ & 4.00 \\
$1 / 64$ & $1.849 E-07$ & 3.99 & $6.192 E-08$ & 3.99 & $1.850 E-07$ & 3.99 & $6.192 E-08$ & 3.99 \\
\hline
\end{tabular}

TABLE 3: Numerical results for the sixth-order CFD method in 2D with $\boldsymbol{\alpha}=\operatorname{Re} \cdot(1,1)$.

\begin{tabular}{|c|c|c|c|c|c|c|c|c|}
\hline \multirow{2}{*}{$h$} & \multicolumn{2}{|c|}{$\operatorname{Re}=1$} & \multicolumn{2}{|c|}{$\operatorname{Re}=10$} & \multicolumn{2}{|c|}{$\operatorname{Re}=10^{2}$} & \multicolumn{2}{|c|}{$\operatorname{Re}=10^{3}$} \\
\hline & Error & Ratio & Error & Ratio & Error & Ratio & Error & Ratio \\
\hline $1 / 4$ & $2.278 E-04$ & & $5.292 E-03$ & & $0.5348 E-01$ & & $0.4837 E-01$ & \\
\hline $1 / 8$ & $3.708 E-06$ & 5.94 & $8.547 E-05$ & 5.95 & $0.2204 E-01$ & 1.28 & $0.1380 E-01$ & 1.81 \\
\hline $1 / 16$ & $5.732 E-08$ & 6.02 & $1.339 E-06$ & 5.99 & $0.1437 E-02$ & 3.94 & $0.3918 E-02$ & 1.82 \\
\hline $1 / 32$ & $9.020 E-10$ & 5.99 & $2.093 E-08$ & 6.00 & $0.1925 E-04$ & 6.22 & $0.1078 E-02$ & 1.86 \\
\hline $1 / 64$ & $1.409 E-11$ & 6.00 & $3.271 E-10$ & 6.00 & $0.3043 E-06$ & 5.98 & $0.2463 E-03$ & 2.13 \\
\hline
\end{tabular}

Example 14. In this example, the diffusive coefficient is regarded as $\kappa\left(x_{1}, \cdots, x_{d}\right)=e^{\sum_{i=1}^{d} x_{i}}$, and then, the forcing function $f$ and the Dirichlet boundary conditions are prescribed to satisfy the exact solution $u\left(x_{1}, \cdots, x_{d}\right)=\prod_{i=1}^{d} \sin \pi x_{i}$. To exhibit the numerical results, we denote two schemes as

(i) Scheme I: (28) with coefficients (30)

(ii) Scheme II: (32) with coefficients (34)

As the computation of the coefficients, we take the following two cases:

(i) Case 1: coefficients (30) and (34) computed analytically

(ii) Case 2: coefficients (30) and (34) computed by the approximation introduced in Remark 3.

The information relevant to maximum errors and convergence rates are listed in Tables 1 and 2 for $2 \mathrm{D}$ and $3 \mathrm{D}$ problems, severally.

From these tables, it offers a brief result that both two schemes are published in almost the same errors with a fourth-order convergence rate. Although the errors in essence were affected by the ways of computing the coefficients, the consequence of convergence rate still remains $O\left(h^{4}\right)$.

Example 15. An example to validate the accuracy of the CFD schemes is proposed in Section 3.2, considering the convection-diffusion equation, constant convection vector coefficients $\boldsymbol{\alpha}$ is regarded as $\mathrm{Re} \cdot \mathbf{e}$, where $\mathbf{e}$ is $d$-dimensional vector with all unit elements. The forcing function $f\left(x_{1}, \cdots\right.$, $x_{d}$ ) and the Dirichlet boundary conditions are prescribed to satisfy the exact solution $u\left(x_{1}, \cdots, x_{d}\right)=\prod_{i=1}^{d} \sin \pi x_{i}$. Re is a representative of the constant conducted to reflect the ratio of the convection to diffusion and simulate the Reynolds number.

For the different Re, the refinement of the mesh size $h$ is undertaken to observe the convergence rate. The maximum errors and the convergence rate of $2 \mathrm{D}$ and $3 \mathrm{D}$ problems are listed as $1 \leq \operatorname{Re} \leq 10^{3}$ in Tables 3 and 4 and in Figure 1, clearly.

It has become apparent that Re is not very large leading to the rapid decrease of errors with the refinement of the mesh 
TABLE 4: Numerical results for the sixth-order CFD method in 3D with $\boldsymbol{\alpha}=\operatorname{Re} \cdot(1,1,1)$.

\begin{tabular}{|c|c|c|c|c|c|c|c|c|}
\hline \multirow{2}{*}{$h$} & \multicolumn{2}{|c|}{$\operatorname{Re}=1$} & \multicolumn{2}{|c|}{$\operatorname{Re}=10$} & \multicolumn{2}{|c|}{$\operatorname{Re}=10^{2}$} & \multicolumn{2}{|c|}{$\operatorname{Re}=10^{3}$} \\
\hline & Error & Ratio & Error & Ratio & Error & Ratio & Error & Ratio \\
\hline $1 / 4$ & $1.195 E-03$ & & $1.047 E-02$ & & $4.286 E-02$ & & $3.798 E-02$ & \\
\hline $1 / 8$ & $1.814 E-05$ & 6.042 & $1.589 E-04$ & 6.043 & $2.303 E-02$ & 0.90 & $1.482 E-02$ & 1.36 \\
\hline $1 / 16$ & $2.924 E-07$ & 5.955 & $2.542 E-06$ & 5.966 & $1.795 E-03$ & 3.68 & $5.782 E-03$ & 1.36 \\
\hline $1 / 32$ & $4.550 E-09$ & 6.006 & $3.985 E-08$ & 5.995 & $2.865 E-05$ & 5.97 & $1.976 E-03$ & 1.55 \\
\hline $1 / 64$ & $7.106 E-11$ & 6.001 & $6.251 E-10$ & 5.994 & $4.547 E-07$ & 5.98 & $4.488 E-04$ & 2.14 \\
\hline
\end{tabular}

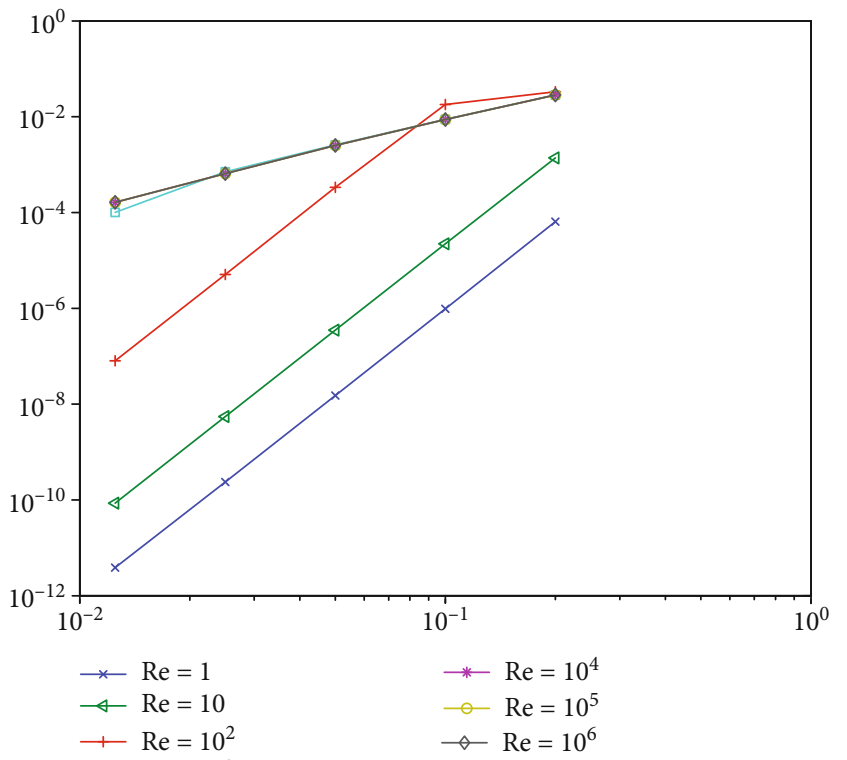

(a)

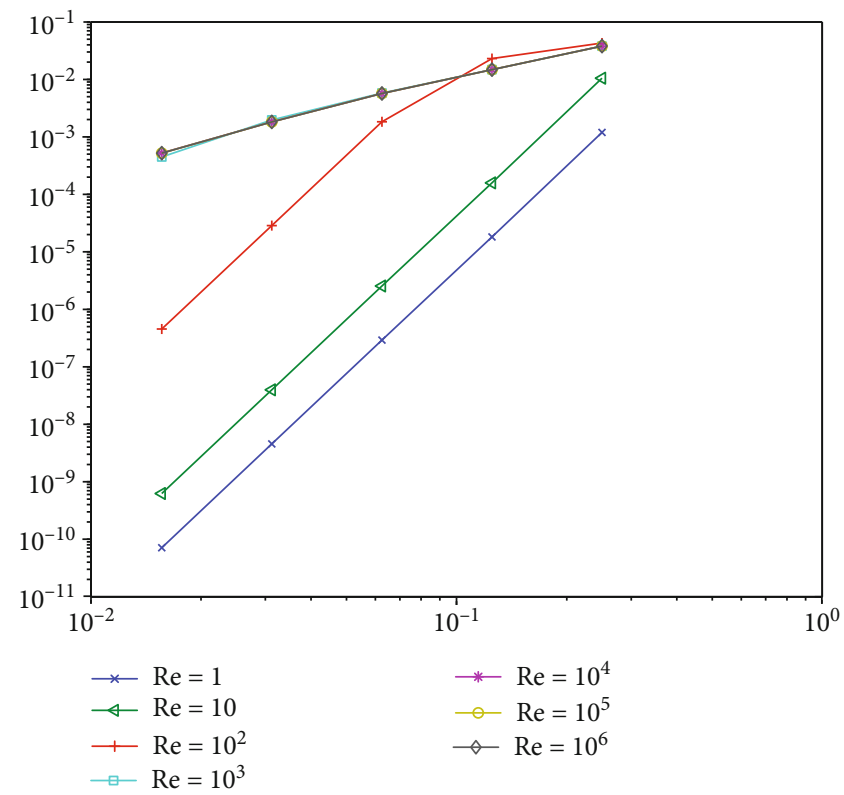

(b)

Figure 1: (a) Test of the sixth-order CFD method in 2D; (b) test of the sixth-order CFD method in 3D.

size $h$ and a sixth-order convergence rate. But with the increase of Re, the computed accuracy is reversely affected. For the large Re, only a second-order convergence rate can be achieved. It is expected that the deterioration of computed accuracy is attributed to a reason of the convectiondominated equation (1), and when the Re increases to be infinite, it approximates a first-order hyperbolic equation.

\section{Conclusions}

Compact finite-difference (CFD) schemes seem to be simple and powerful ways contributing to a deeper understanding of high accuracy and low computational cost. Compared with the traditional explicit finite-difference schemes of the same-order, the effects of compact schemes are significantly more accurate with the benefit of smaller stencil sizes. In the experimented present work, the fourth-order CFD schemes generate fresh insight into a general formulation of convection-diffusion equations with variable convective coefficients and elliptic diffusion equations with variable diffusive coefficients in any dimension. When the convective coefficients are constants, the sixth-order CFD schemes provide an important opportunity to solve convection-diffusion problems. Numerical results of preliminary work show the efficiency of the proposed CFD schemes. A significant analysis and discussion on similar approaches may be extended to elliptic diffusion problems with positive diffusive tensor in any dimension and are under investigation.

\section{Data Availability}

The data used to support the findings of this study are available from the corresponding author upon request.

\section{Conflicts of Interest}

The authors declare that there are no conflicts of interests regarding the publication of this paper.

\section{Acknowledgments}

The first author acknowledges financial support from the Wuhan Business University Doctoral Foundation under Grant 2019KB003. 


\section{References}

[1] B. P. Leonard, "A stable and accurate convective modelling procedure based on quadratic upstream interpolation," Computer Methods in Applied Mechanics and Engineering, vol. 19, no. 1, pp. 59-98, 1979.

[2] A. Golbabai and M. M. Arabshahi, "A numerical method for diffusion-convection equation using high-order difference schemes," Computer Physics Communications, vol. 181, no. 7, pp. 1224-1230, 2010.

[3] W. Y. Liao, "A compact high-order finite difference method for unsteady convection-diffusion equation," International Journal for Computational Methods in Engineering Science and Mechanics, vol. 13, no. 3, pp. 135-145, 2012.

[4] Y. Ma and Y. B. Ge, "A high order finite difference method with Richardsonextrapolation for 3D convection diffusion equation," Applied Mathematics Compututation, vol. 215, no. 9, pp. 3408-3417, 2010.

[5] Z. F. Tian and S. Q. Dai, "High-order compact exponential finite difference methods for convection diffusion type problems," Journal of Computational Physics, vol. 220, no. 2, pp. 952-974, 2007.

[6] P. Freitas, J. Mao, and I. Salavessa, "Spherical symmetrization and the first eigenvalue of geodesic disks on manifolds," Calculus of Variations and Partial Differential Equations, vol. 51, no. 3-4, pp. 701-724, 2014.

[7] J. Wang, W. J. Zhong, and J. Zhang, “A general meshsize fourth-order compact difference discretization scheme for 3D Poisson equation," Applied Mathematics Compututation, vol. 183, no. 2, pp. 804-812, 2006.

[8] S. Y. Zhai, X. Feng, and Y. He, "A family of fourth-order and sixth-order compact difference schemes for the threedimensional Poisson equation," Journal of Scientific Computing, vol. 54, no. 1, pp. 97-120, 2013.

[9] M. Nabavi, M. H. K. Siddiqui, and J. Dargahi, “A new 9-point sixth-order accurate compact finite-difference method for the Helmholtz equation," Journal of Sound and Vibration, vol. 307, no. 3-5, pp. 972-982, 2007.

[10] X. Feng, "A high-order compact scheme for the onedimensional Helmholtz equation with a discontinuous coefficient," International Journal of Computer Mathematics, vol. 89, no. 5, pp. 618-624, 2012.

[11] X. Feng, Z. L. Li, and Z. H. Qiao, "High order compact finite difference schemes for the Helmholtz equation with discontinuous coefficients," Journal of Computational Mathematics, vol. 29, pp. 324-340, 2018.

[12] N. Mohamed, S. A. Mohamed, and L. F. Seddek, "Exponential higher-order compact scheme for 3D steady convectiondiffusion problem," Applied Mathematics and Computation, vol. 232, pp. 1046-1061, 2014.

[13] H. W. Sun and J. Zhang, "A high-order finite difference discretization strategy based on extrapolation for convection diffusion equations," Numerical Methods for Partial Differential Equations, vol. 20, no. 1, pp. 18-32, 2004.

[14] D. Del Sarto and E. Deriaz, "A multigrid AMR algorithm for the study of magnetic reconnection," Journal of Computational Physics, vol. 351, pp. 511-533, 2017.

[15] S. Bilbao and B. Hamilton, "Higher-order accurate two-step finite difference schemes for the many-dimensional wave equation," Journal of Computational Physics, vol. 367, pp. 134-165, 2018.
[16] M. M. Gupta, R. P. Manohar, and J. W. Stephenson, “A single cell high order scheme for the convection-diffusion equation with variable coefficients," International Journal for Numerical Methods in Fluids, vol. 4, no. 7, pp. 641-651, 1984.

[17] J. Zhang, "An explicit fourth-order compact finite difference scheme for three-dimensional convection-diffusion equation," Communications in Numerical Methods in Engineering, vol. 14, no. 3, pp. 209-218, 1998. 